\title{
Visibility of transparent objects in the eye by retroillumination
}

\author{
NICHOLAS BROWN \\ Moorfields Eye Hospital, City Road, London
}

Retroillumination is performed at the slit-lamp with the binocular microscope focused upon the object which is illuminated from behind by the slit-lamp beam reflected off some structure posterior to the object (Vogt, 1930). Objects in the cornea and lens are observed in this way and the reflectors used are the iris, the lens, or the fundus. The use of the ophthalmoscope for retroillumination of the cornea is also described (Chandler, 1945).

Objects are visible because they interfere with the emergent light from the eye by obstruction, refraction, respersion (Berliner, 1943), and reflection. Transparent objects are visible by retroillumination when the refractive index of the object is above or below that of the surrounding medium. The property mainly responsible is refraction, but reflection also plays some part. The observed appearance changes with the direction of the retroillumination and with the refractive nature of the object. From the appearance of an object under varying retroillumination conditions, conclusions can be drawn about the refractive nature of the object. Although a variety of combinations of shapes and refractive indices are possible in the object, these can be resolved into only two observable effects:

(1) The object acting as a diverging refractor (lens or cylinder).

(2) The object acting as a converging refractor (lens or cylinder).

Clinical examples are shown in macrophotographs made by the technique previously described (Brown, 1970). Photographs are shown for comparison of a diverging lens (plano-concave) and of a converging lens (plano-convex) (Fig. 2). A model has been constructed to resemble objects found in the eye. The model has two spherical objects imbedded in a clear medium (gelatin); one object of low refractive index (air) which acts as a diverging lens, and one of high refractive index (liquid paraffin) which acts as a converging lens. Photographs are shown of the model under varying conditions of illumination (Figs 3, 4, and 5).

\section{Methods of retroillumination}

MARGINAL RETROILlumination (Graves, i924)

This is produced by illuminating half the field behind the object, with the object placed in front of the conjunction between light and dark areas. This can be achieved by using the edge of the decentred slit beam, or by using a natural boundary such as the pupil margin.

The distribution of light within the object is seen to take one of two forms:

(r) Unreversed: the distribution is the same as that of the background.

(2) Reversed: the distribution is opposite to that of the background. 
The unreversed effect is produced by an object acting as a diverging refractor and the reversed effect by a converging refractor, provided that its focal length is shorter than the distance between the object and the area of retroillumination. The optical explanation of the effect is shown in Fig. IA.

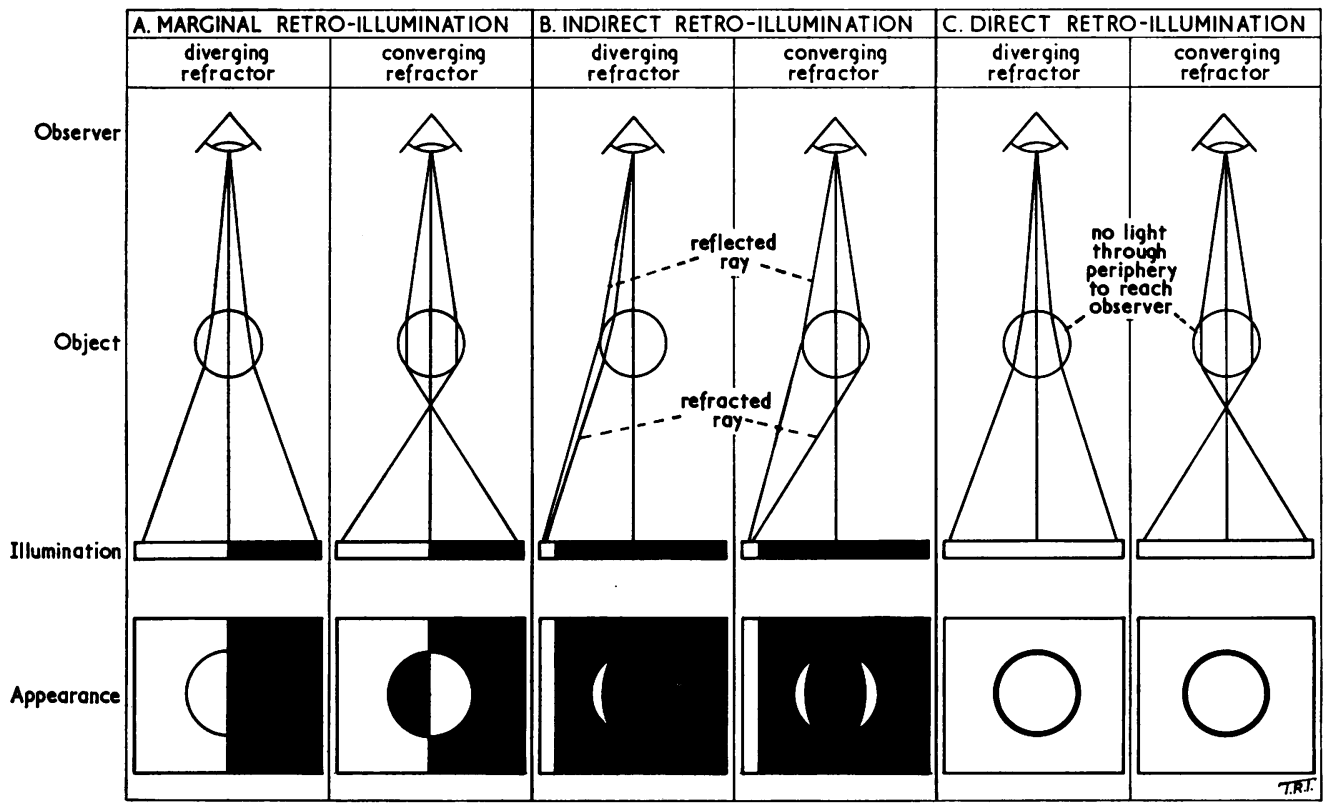

FIG. I Optics of retroillumination

2

3
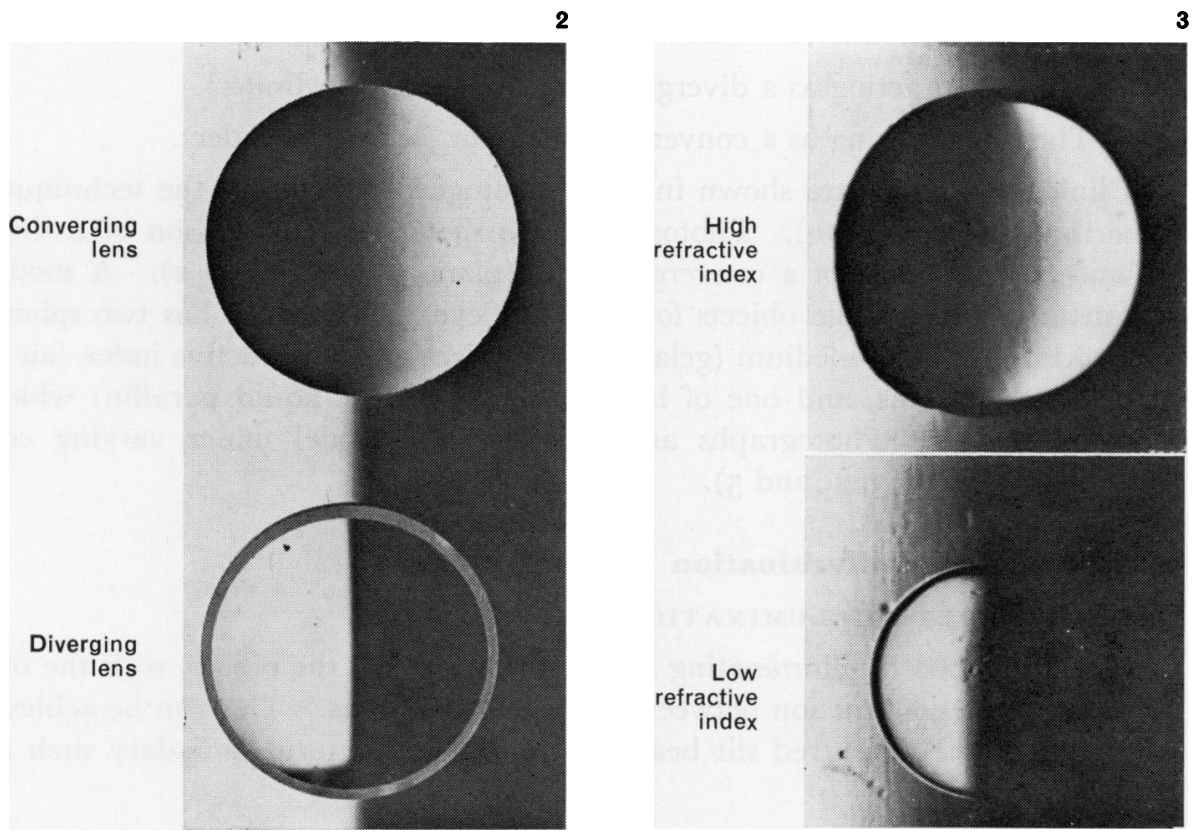

FIG. 2 Lenses. The diverging lens shows the unreversed effect. The converging lens shows the reversed effect

FIG. 3 Model. Marginal retroillumination. The low refractive index object shows the unreversed effect. The high refractive index object shows the reversed effect 

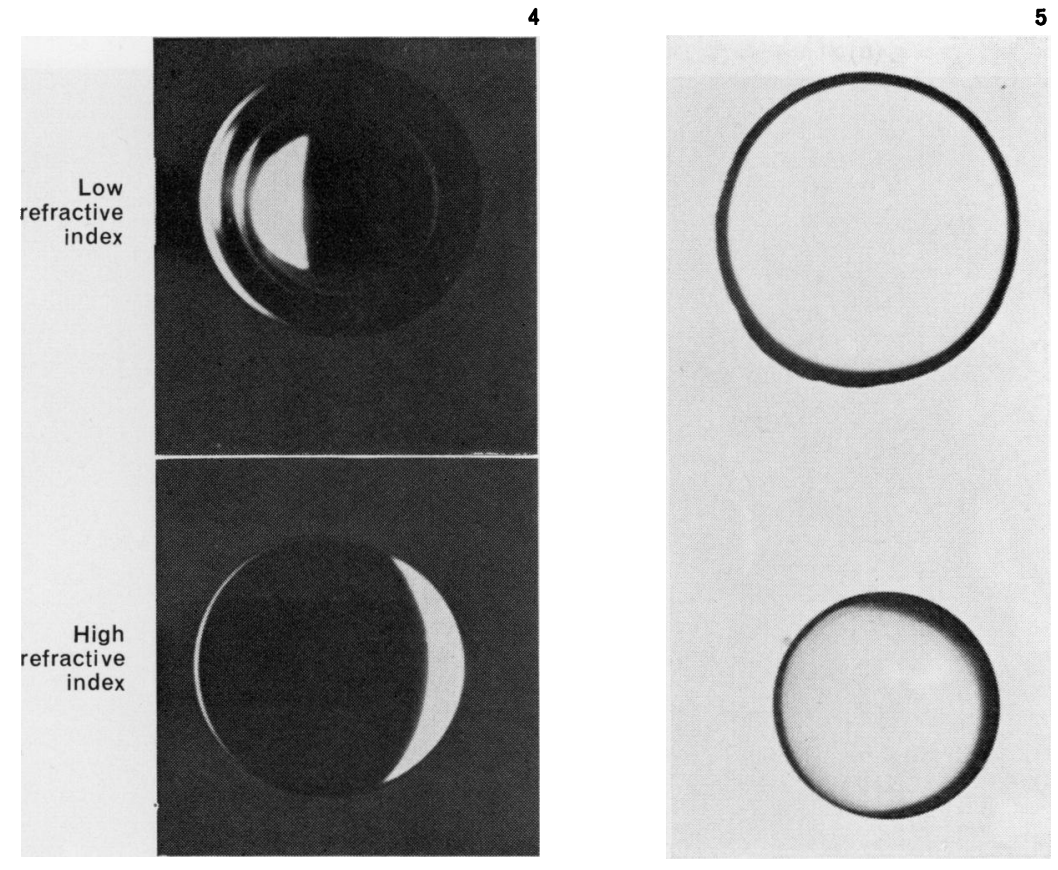

FIG. 4 Model. Indirect retroillumination. The low refractive index object shows refracted and reflected light on one side. The high refractive index object shows reflected and refracted light on opposite sides

FIG. 5 Model. Direct retroillumination. Both objects show dark edges

In the photograph of the lenses (Fig. 2) the unreversed effect is seen in the diverging lens and the reversed effect in the converging lens. In the model (Fig. 3), the unreversed effect is seen in the object of low refractive index and the reversed effect in the object of high refractive index.

Clinical examples of spherical objects showing the unreversed effect are shown in Figs 6 and 7 and the reversed effect in Figs 8 and 9. Cylindrical objects showing the unreversed effect are shown in Figs Io and I I and the reversed effect in Figs 12 and I 3.

Vogt (1930) recognized that cystic objects reversed light, and he used as an example a photograph of rain drops upon a window. Graves (1924) was able to separate the appearances into unreversed and reversed effects and suggested that this was due to the refractive nature of the object.

INDIRECT RETROILLUMINATION (Berliner, I943)

This is produced by displacing the retroillumination to one side of the object, so that the object is observed against a dark background. When this is done the unreversed or reversed effects become limited to crescents in spherical objects and to the marginal region in cylindrical objects. These effects may be seen alone, and also in conjunction with surface reflections (described below) as is seen in the photographs of the model (Fig. 4). The optics of indirect retroillumination are shown in Fig. I B.

Clinical examples showing crescents are seen with the unreversed effect in Figs 6 and I 4, and with the reversed effect in Fig. 8. A cylindrical object showing the reversed effect limited to the marginal region is shown in Fig. I3. 
(6)

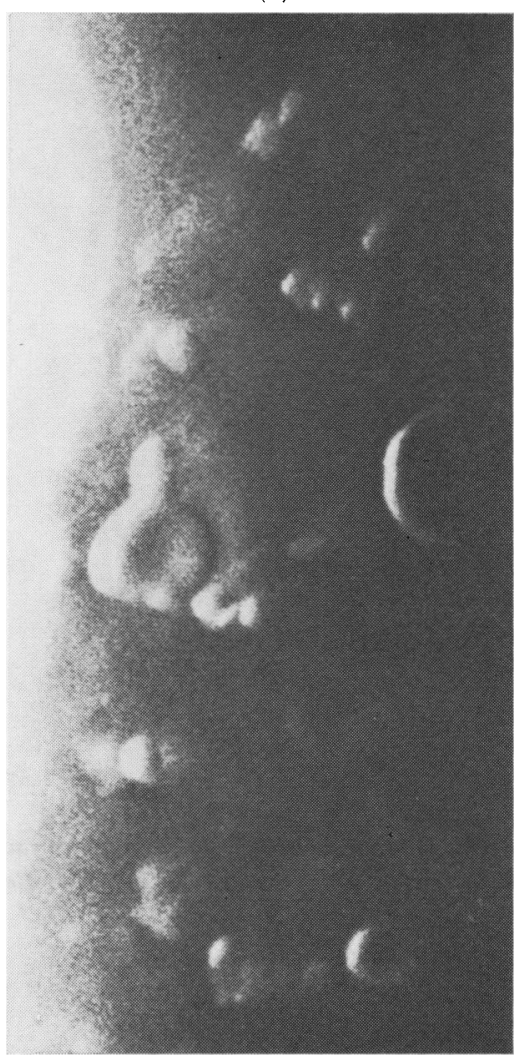

(7)

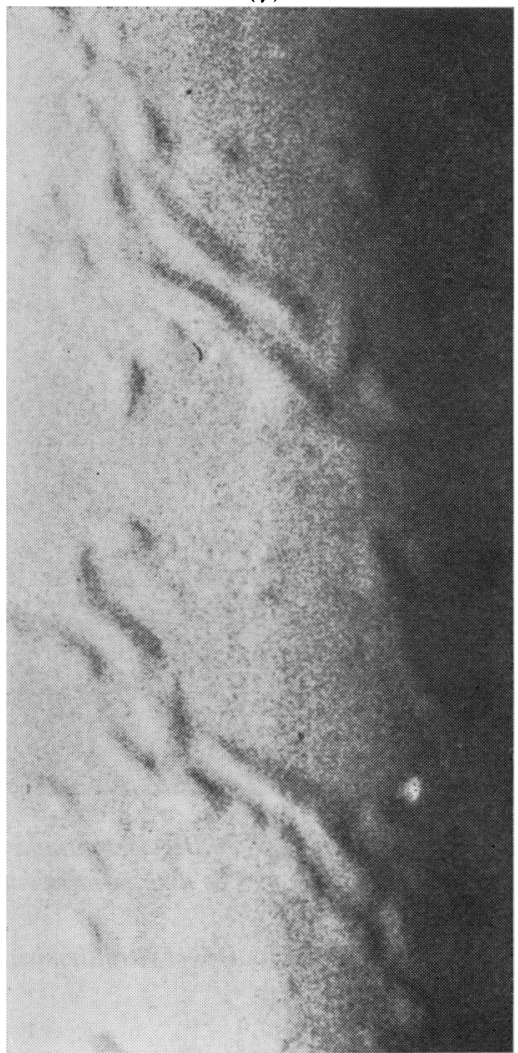

F IG. 6 Intraepithelial cysts. Marginal und indirect retroillumination unreversed effect and reflections

FIG. 7 Net-like dystrophy. Marginal retroillumination. Unreversed effect

Surface reflections occur with both spherical and cylindrical objects observed by indirect retroillumination. The reflection occurs on the same side as the illumination, so that the reflection contributes to the crescent of the unreversed effect, but is on the opposite side to the reversed effect. The converging type of spherical object therefore shows two bright crescents, one on each side. The optics of reflections are shown in Fig. I B. With the low refractive index object, this is by total internal reflection within the denser medium, and with the high refractive index object it is a surface reflection off the object.

Reflections are seen in the photograph of the model (Fig. 4), and in clinical examples of spherical objects (Figs 6 and 8), and of a cylindrical object (Fig. I3).

DIRECT RETROILLUMINATION

The object is illuminated by an evenly lit background directly behind it. The object is then distinguished from the background by a dark edge which is present for both converging and diverging objects. The luminosity of the central parts of the object appear similar to the background illumination. In practice it is not easy to produce a perfectly evenly lit background, so that the dark edge effect is usually combined with the unreversed or reversed effects. 


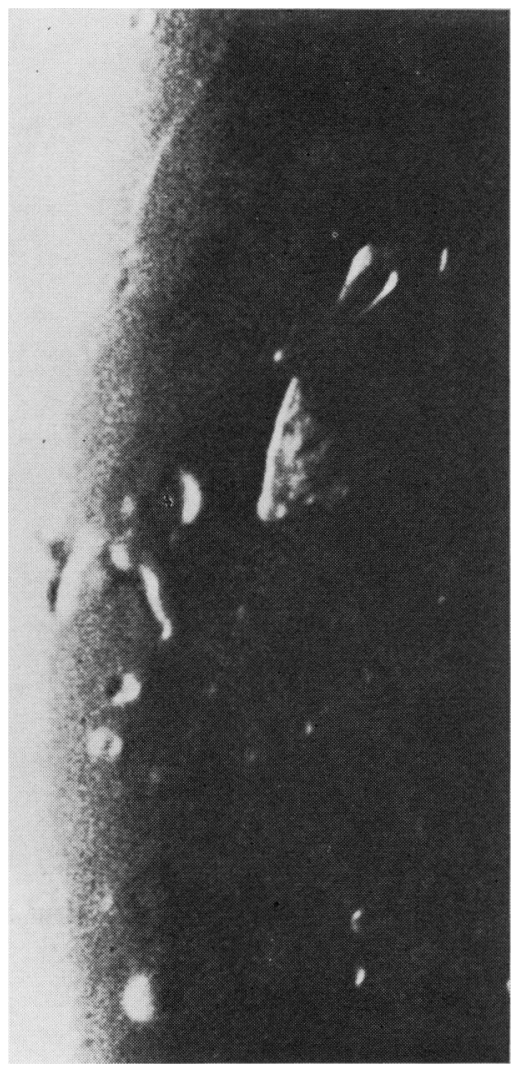

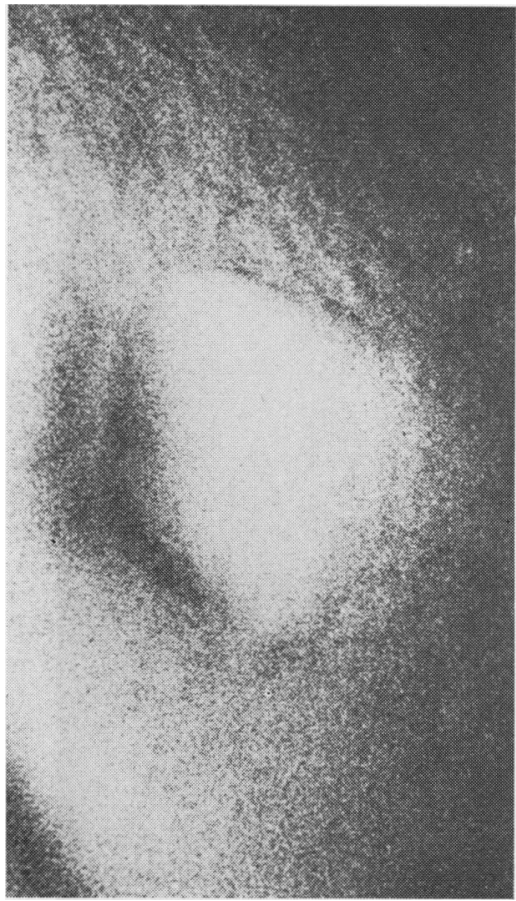

FIG. 9 Epithelial bulla. Marginal retroillumination. Reversed effect

FIG. 8 Intraepithelial cysts. Marginal and indirect retroillumination. Reversed effect and reflections

The dark edge is accounted for by rays being unable to pass through the periphery of an object, acting as a thick lens to reach the observer (Fig. IC).

The dark edge is shown by the spherical objects in the model (Fig. 5); the clinical example in which it is best seen is Fig. I 5 .

\section{Discussion}

The effects seen in transparent objects are resolvable into:

(I) Objects acting as a diverging refractor.

(2) Objects acting as a converging refractor.

The ways in which these can be recognized have been described above.

The shape of the object can be confidently determined in some cases with additional clues from the appearance by focal illumination and from the disturbance of the tear film and of the specular reflex when present. When these clues are not available the shape of the object remains speculative.

The cornea is a substance of relatively high refractive index I.376 (Matthiessen, I 89 I) compared to the aqueous of index 1.336 (Duke-Elder, 1938). The corneal epithelium has a higher refractive index than the stroma of 1.416 (Fischer, 1927).

The following types of object have refractive properties. 


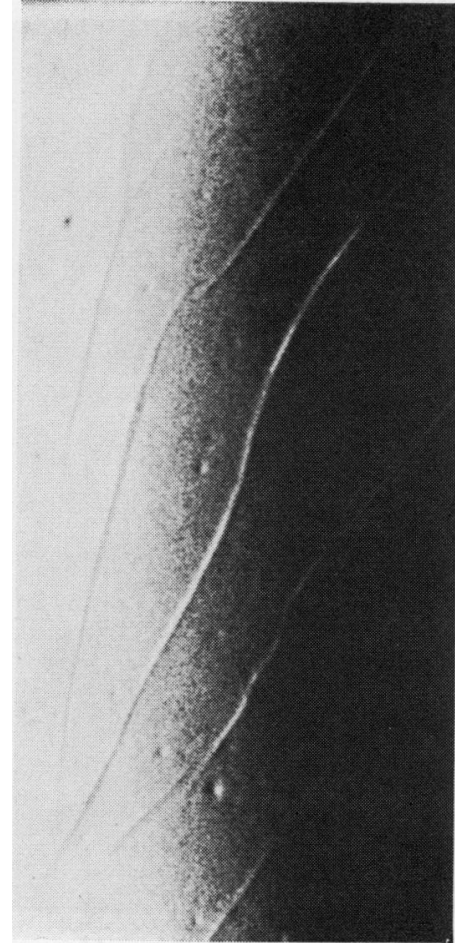

FIG. Io Finger print lines. Marginal retroillumination. Unreversed effect

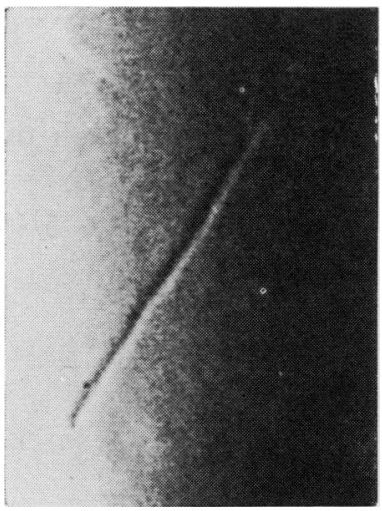

FIG. I2 Stromal microfilaria. Marginal retroillumination. Reversed effect

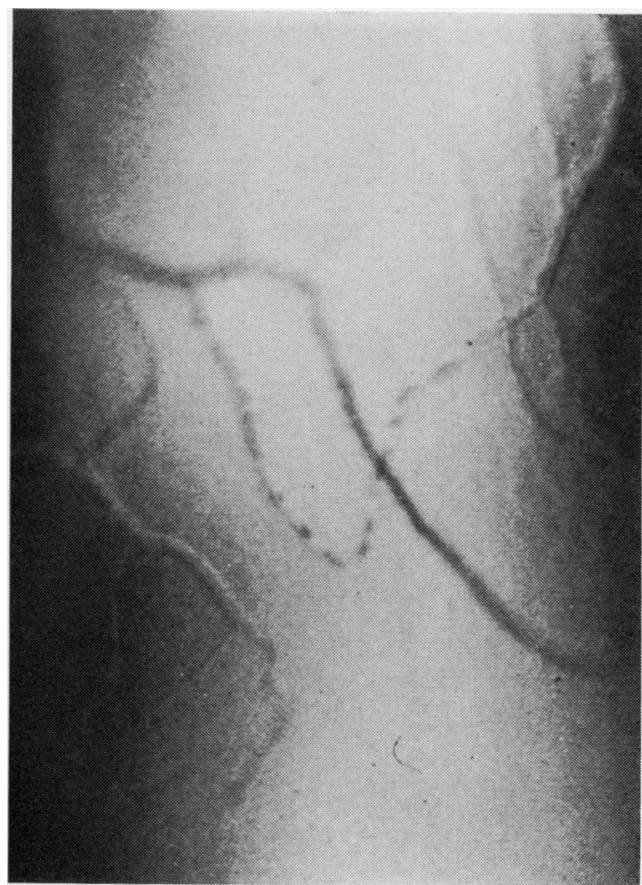

FIG. I I Stromal blood vessels. Marginal retroillumination. Unreversed effect

FIG. 14 Lens cyst. Indirect retroillu-

mination. Unreversed effect
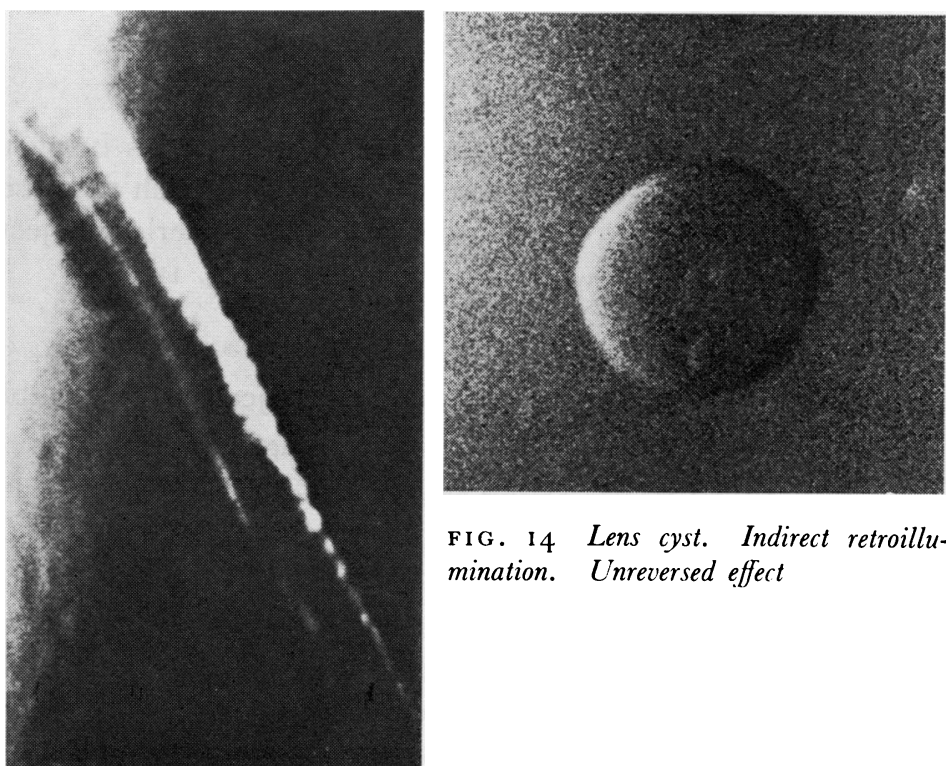

FIG. I3 Descemet's splits. Marginal and indirect retroillumination 


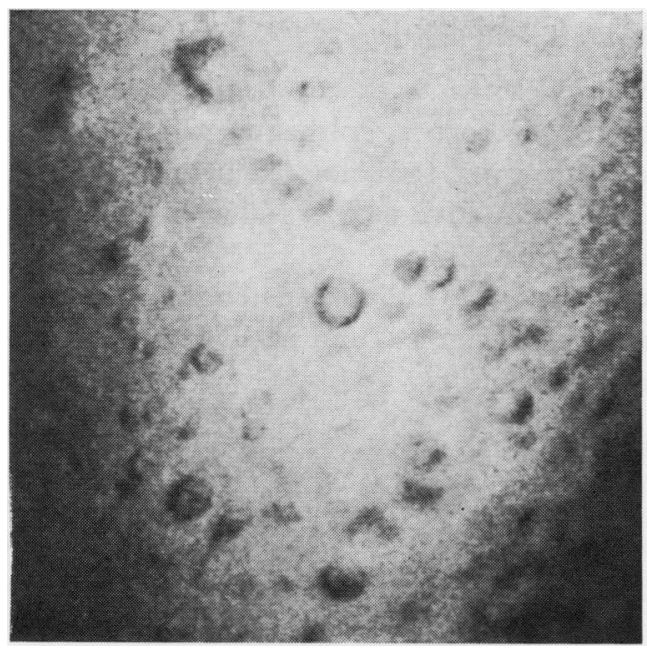

FIG. I5 Intraepithelial cysts. Direct retroillumination. Dark edges

Object interfering with a surface

Objects which project from the anterior or posterior surfaces of the cornea into the air or aqueous respectively act as converging refractors since they project into regions of lower refractive index. Objects of this type are visible by focal illumination and disturb the specular reflex and also the tear film when anterior.

The shapes which these objects may take are shown in Fig. 16A. Clinical examples are epithelial bullae (Fig. 9), endothelial colloid bodies, and the so-called Descemet's splits (Fig. I3), which project into the aqueous.

FIG. I6 Shape of objects visible by retroillumination

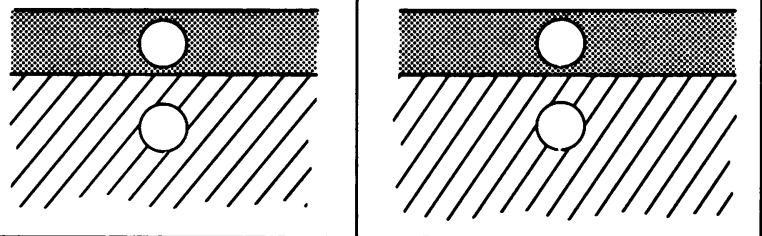


Object projecting into a tissue of different refractive index

This type of object can occur wherever two adjacent tissues have different refractive indices, in particular at the junction of the corneal epithelium with Bowman's membrane. The objects act as diverging or converging refractors, depending upon whether they project into a zone of higher or lower refractive index. Clues to their shape are usually not available from other methods of examination.

The shapes which these objects may take are shown diagrammatically in Fig. I6B. Clinical examples are the fingerprint line (Fig. 10) and net-like dystrophy (Fig. 7), which both behave as diverging refractors.

Objects wholly within a tissue of different refractive index

This type of object is seen within the corneal epithelium, in the corneal stroma, and in the $\frac{\omega}{O}$ lens. When the refractive index of the object is lower than that of the surrounding tissue it acts as a diverging refractor, and when the refractive index of the object is higher $G$ it acts as a converging refractor.

The shape of this type of object is shown diagramatically in Fig. $16 \mathrm{c}$ and is imitated $\stackrel{G}{V}$ by the model. Clinical examples are intraepithelial cysts (Figs 6, 8, and I5), and blood $\frac{}{2}$ vessels and microfilariae in the corneal stroma (Figs II and I2), and also the lens cyst $\rightarrow$ (Fig. 14).

If the object of this type is large enough to disturb the surface it then acts as an object $\frac{6}{2}$ interfering with a surface such as the corneal bulla (Fig. 9).

I wish to thank Prof. Barrie R. Jones for giving me access to his patients and Mr. T. R. Tarrant of the Institute of Ophthalmology who prepared the diagrams.

\section{References}

BerLiner, M. L. (1943) "Biomicroscopy of the Eye", vol. I, p. 79. Hoeber, New York BRown, N. (1970) Brit. J. Ophthal., 54, 697 CHANDLER, P. A. (1945) Amer. 7. Ophthal., 28, 355 DUKE-ELDER, s. (1938) “Textbook of Ophthalmology”, vol. I, p. 744. Kimpton, London FISGHER (1927) Ber. dtsch. ophthal. Ges., 46, 429

GRAVEs, B. (1924) Brit. F. Ophthal., 8, 502

matthiessen, L. (1891) "Die neueren Fortschritte in unserer Kenntnis von dem optischen Baue des Auges der Wirbeltiere". Voss, Hamburg voGt, A. (1930) "Lehrbuch und Atlas der Spaltlampenmikroskopie des lebenden Auges, 2nd ed.

Erster Teil: Technik und Methodik Hornhaut und Vorderkammer”, p. 2 I. Springer, Berlin 